\title{
Locked and Unlocked Chains of Planar Shapes
}

\author{
Robert Connelly • Erik D. Demaine • Martin L. Demaine • Sándor P. Fekete • \\ Stefan Langerman • Joseph S.B. Mitchell • Ares Ribó • Günter Rote
}

Received: 9 May 2007 / Revised: 3 May 2010 / Accepted: 9 May 2010 / Published online: 28 May 2010 (C) Springer Science+Business Media, LLC 2010

\begin{abstract}
We extend linkage unfolding results from the well-studied case of polygonal linkages to the more general case of linkages of polygons. More precisely, we con-
\end{abstract}

\begin{abstract}
A preliminary extended abstract of this paper appeared in Proceedings of the 22nd Annual ACM Symposium on Computational Geometry, June 2006, [7]. R. Connelly is supported in part by NSF grant DMS-0209595. E. Demaine is supported in part by NSF grant CCF-0347776 and DOE grant DE-FG02-04ER25647. S. Langerman is Chercheur qualifié du FNRS. J. Mitchell is supported in part by NSF (CCF-0431030, CCF-0528209, CCF-0729019), Metron Aviation, and NASA Ames.
\end{abstract}

R. Connelly $(\bowtie)$

Department of Mathematics, Cornell University, Ithaca, NY 14853, USA

e-mail: connelly@math.cornell.edu

E.D. Demaine · M.L. Demaine

MIT Computer Science and Artificial Intelligence Laboratory, 32 Vassar St., Cambridge, MA 02139, USA

E.D. Demaine

e-mail: edemaine@mit.edu

M.L. Demaine

e-mail: mdemaine@mit.edu

S.P. Fekete

Department of Computer Science, Braunschweig University of Technology, Mühlenpfordtstr. 23, 38106 Braunschweig, Germany

e-mail: s.fekete@tu-bs.de

S. Langerman

Chercheur qualifié du FNRS, Département d'informatique, Université Libre de Bruxelles,

ULB CP212, Bruxelles, Belgium

e-mail: Stefan.Langerman@ulb.ac.be

J.S.B. Mitchell

Department of Applied Mathematics and Statistics, Stony Brook University, Stony Brook, NY 11794-3600, USA

e-mail: jsbm@ams.sunysb.edu 
sider chains of nonoverlapping rigid planar shapes (Jordan regions) that are hinged together sequentially at rotatable joints. Our goal is to characterize the families of planar shapes that admit locked chains, where some configurations cannot be reached by continuous reconfiguration without self-intersection, and which families of planar shapes guarantee universal foldability, where every chain is guaranteed to have a connected configuration space. Previously, only obtuse triangles were known to admit locked shapes, and only line segments were known to guarantee universal foldability. We show that a surprisingly general family of planar shapes, called slender adornments, guarantees universal foldability: roughly, the distance from each edge along the path along the boundary of the slender adornment to each hinge should be monotone. In contrast, we show that isosceles triangles with any desired apex angle $<90^{\circ}$ admit locked chains, which is precisely the threshold beyond which the slender property no longer holds.

Keywords Adornments - Carpenter's rule · Hinged dissections · Locked chains · Monotone motion · Polygonal chains · Rigidity · Slender adornments · Unfolding chains

\section{Introduction}

In this paper, we explore the motion-planning problem of reconfiguring or folding a hinged collection of rigid objects from one state to another while avoiding self-intersection. This general problem has been studied since the beginnings of the motion-planning literature when Reif [23] proved that deciding reconfigurability of a "tree" of polyhedra, amidst fixed polyhedral obstacles, is PSPACE-hard. This result has been strengthened in various directions over the years, although the cleanest versions were obtained only very recently: deciding reconfigurability of a tree of line segments in the plane, and deciding reconfigurability of a chain of line segments in $3 \mathrm{D}$, are both PSPACE-complete [3]. This result is tight in the sense that deciding reconfigurability of a chain of line segments in the plane is easy, in fact, trivial: the answer is always yes [9].

These results illustrate a rather fine line in reconfiguration problems between computationally difficult and computationally trivial. The goal of our work is to characterize what families of planar shapes and hingings lead to the latter outcome, a universality result that reconfiguration is always possible. The only known example of such a result, however, is the family of chains of line segments, and that problem was unsolved for about 25 years [9]. (A chain is a sequence of line segments joined endto-end that are disjoint except for consecutive endpoints, where they are hinged. It is

A. Ribó · G. Rote

Institut für Informatik, Freie Universität Berlin, Takustraße 9, 14195, Berlin, Germany

A. Ribó

e-mail: ribo@inf.fu-berlin.de

G. Rote

e-mail: rote@inf.fu-berlin.de 
Fig. 1 Simple examples of locked chains of triangles. (a) A locked chain of three triangles. (b) A locked chain of equilateral triangles of different sizes. The gaps should be tighter than drawn

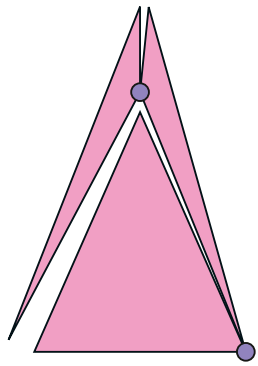

(a)

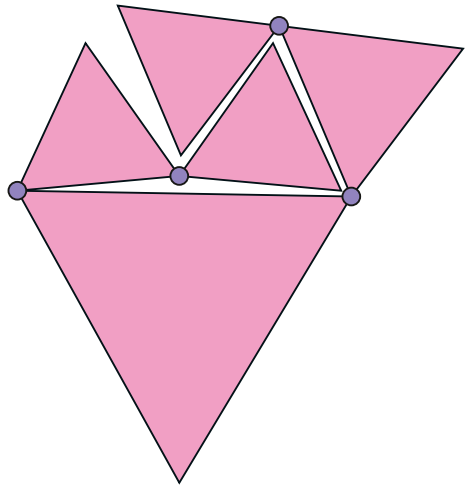

(b)

called an open chain when the first line segment is not joined to the last and closed when it is joined to the last segment in a closed cycle.) Even small perturbations to the problem, such as allowing a single point where three line segments join, leads to locked examples where reconfiguration is impossible [8].

What about chains of shapes other than line segments? It is easy to see that a shape tucked into a "pocket" of a nonconvex shape immediately makes trivial locked chains with two pieces. Back in January 1998, the third author showed how to simulate this behavior with convex shapes, indeed, just three triangles; see Fig. 1(a). This example has circulated throughout the years to many researchers (including the authors of this paper) who have asked about chains of $2 \mathrm{D}$ shapes. The only really unsatisfying feature of the example is that some of the angles are very obtuse. But with a little more work, one can find examples with acute angles, indeed, equilateral triangles, albeit of different size; see Fig. 1(b). What could be better than equilateral triangles?

It is therefore reasonable to expect, as we did for many years, that there is no interesting class of shapes, other than line segments, with a universality result-essentially all other shapes admit locked chains. We show in this paper, however, that this guess is wrong.

We introduce a family of shapes, called slender adornments, and prove that all open chains, made up of arbitrarily many different shapes from this family, can be universally reconfigured between any two states. Indeed, we show that these chains have a natural canonical configuration, analogs of the straight configuration of an open chain. Our result is based on the existence of "expansive motions," proved in [9]. Our techniques build on the theory of unfolding chains of line segments, substantially generalizing and extending the results from that theory. Indeed, the results in this paper essentially piggy-back onto the results of [9] or any of the other results and algorithms such as $[24,25]$ that provide a continuous expansive motion of the base chain. Our results go far beyond what we thought was possible (until recently). As part of the methods that we describe here, we also consider discrete expansive motions of the base chain that do not necessarily come from a continuous expansive motion. (A discrete expansion of a chain $C$ is simply another corresponding chain $C^{\prime}$ such that if $x$ and $y$ are two points in $C$, the distance between corresponding points $x^{\prime}$ and $y^{\prime}$ is not smaller than the distance between $x$ and $y$.) In that case if all the slen- 


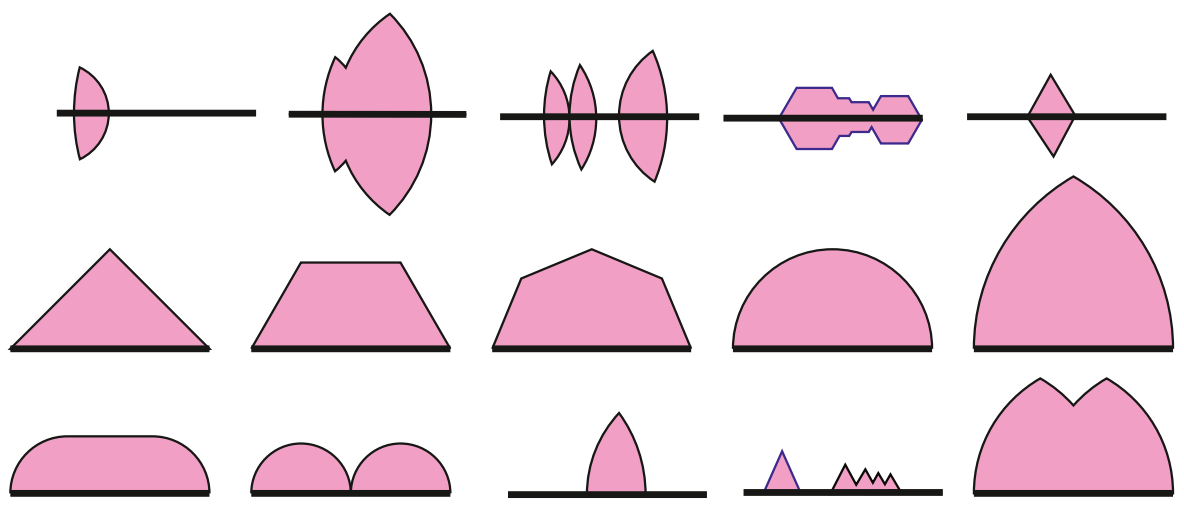

Fig. 2 Examples of slender adornments. The base is drawn bold. The examples in the top row are symmetric. Any two of these examples can be glued together along a common base so that the union also becomes a slender adornment

der adornments are symmetric under the reflection about the line of the base chain, then any expansive discrete motion of the base chain will have the property that the attached adornments will not overlap. It turns out that the continuous case, when the adornments are not necessarily symmetric, follows from the discrete symmetric case.

The family of slender adornments has several equivalent definitions. The key idea is to distinguish the two hinge points on the boundary of the shape connecting to the adjacent shapes in the chain and view the shape as an adornment to the line segment connecting those two hinges, called the base. This view is without loss of generality but provides additional information relating the shapes and how they are attached to neighbors, which turns out to be crucial to obtaining a universality result. An adornment is slender if the distance from either endpoint of the base to a point moving along one side of the adornment changes monotonically. If the boundary curve, defining the adornment, is sufficiently smooth, it is slender if and only if every inward normal of the shape hits the base. Equivalently, an adornment is slender if it is the union, in each half-plane having the base as a boundary, of the intersection of pairs of disks centered at the two endpoints of the base. For the first and last edge of an open chain, there may be some freedom in defining the base.

Slender adornments are quite general. Figure 2 shows several examples of slender adornments. These examples are themselves slender adornments, but also any pair can be joined along their bases so that the union makes another slender adornment. Our results imply that one can take any of these slender adornments, link the bases together into an open chain in any way that the chain does not self-intersect, and the resulting chain can be unfolded without self-intersection to a straight configuration, and thus the chain can be folded without self-intersection into every configuration.

We also demonstrate the tightness of the family of slender adornments by giving examples of locked chains of shapes that are not slender. Specifically, we show that, for any desired angle $\theta<90^{\circ}$, there is a locked chain of isosceles triangles with apex angle $\theta$. This is precisely the family of isosceles-triangle adornments that are not slender. Thus, for chains of triangles, obtuseness is really desirable, contrary to our intuition from Fig. 1(a): the key is that the apex angle opposite the base (in the 
Fig. 3 Hinged dissection of square to equilateral triangle, described by Dudeney [17]. Different shades show different folded states (overlapping slightly)

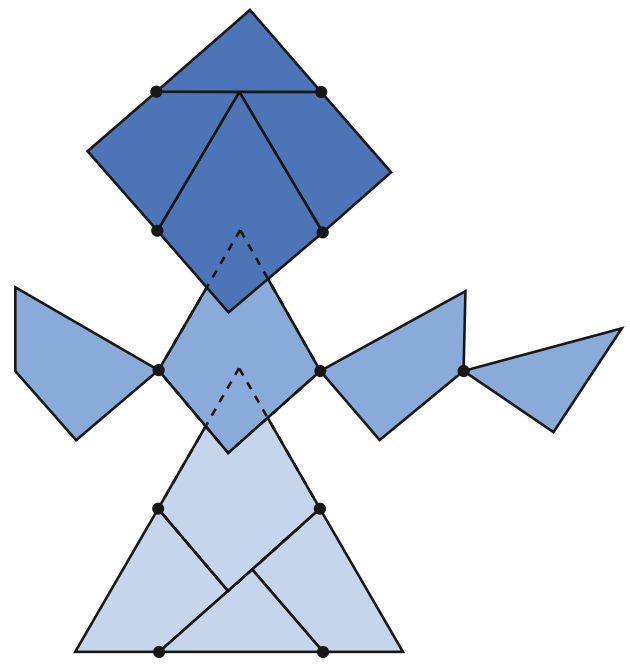

adornment view) be nonacute, not any other angle. The proof that our examples are locked uses the self-touching theory developed for trees of line segments in [8].

\subsection{Motivation}

Hinged collections of rigid objects have been studied previously in many contexts, particularly robotics. One recent body of algorithmic work by Cheong et al. [14] considers how chains of polygonal objects can be immobilized or grasped by a robot with a limited number of actuators. Grasping is a natural first step toward robotic manipulation, but the latter challenge requires a better understanding of reconfigurability. This paper offers the first theoretical underpinnings for reconfiguration of chains of rigid objects (other than line segments).

Another potential application is to continuous folding of hinged dissections. Hinged dissections are chains or trees of polygons that can be reconfigured into two or more self-touching configurations with desired silhouettes. For example, Fig. 3 shows a classic hinged dissection from 1902 of a square into an equilateral triangle of the same area. Many general families of hinged dissections have been established in the recent literature $[5,15,16,18,19]$. One problem not addressed in this literature, however, is whether the reconfigurations can actually be executed without selfintersection, as in Fig. 3. Our results provide potential tools, previously lacking, for addressing this problem. While hinged dissections have frequently been considered in recreational contexts, they have recently found applications in nanomanufacturing [22] and reconfigurable robotics [16].

\subsection{Outline}

This paper is organized as follows. Section 2 defines the model and slender adornments more precisely and proves several basic properties. Section 3 describes the case where each adornment is symmetric about its base and is important for proving, 
in Sect. 4, that simple chains of slender adornments can always be unfolded so that the base is convex or straight. In Sect. 5 we discuss the situation when the adornments are permitted to overlap. Section 6 describes our examples of locked chains of isosceles triangles, including the necessary background from self-touching trees. The conclusion (Sect. 7) includes several closing remarks.

The results of this paper have been reviewed and sketched in the survey [11, Sect. 4], which cites this paper (in its full form) as the source of the results and the proofs.

\section{Slender Adornments}

This section provides a formal statement of the objects we consider-adorned chains consisting of slender adornments - and proves several basic results about them.

\subsection{Adorned Chains}

Our object of study is a chain of nonoverlapping rigid planar shapes (Jordan regions) that are hinged together sequentially at rotatable joints. Another way to view such a chain is to consider the underlying polygonal chain, the core, of line segments connecting successive joints. (For an open chain, there is some freedom in choosing the endpoints for the first and the last bar of the chain.) On the one hand, these line segments can be viewed as bars that move rigidly with the shapes to which they belong. On the other hand, the shapes can be viewed as "adornments" to the bars of an underlying polygonal chain. This view leads to the concept of an "adorned polygonal chain," which we now proceed to define more precisely.

An adornment is a simply connected compact region in the plane, called the shape, together with a line segment $x y$ connecting two boundary points, called the base. There are two boundary arcs from $x$ to $y$ that enclose the shape, called sides. We require the base to be contained in the shape; i.e., the base must be a chord of the shape.

We say that two distinct adornments overlap when some point of one adornment lies in the interior of the other, and we insist that the relative interiors of the base chains be disjoint. Thus, the bases of two shapes are not allowed to touch except at common hinges of the polygonal chain. An adorned polygonal chain is a set of nonoverlapping adornments whose bases form a polygonal chain. We permit the shapes to touch on their boundary and to slide along each other.

For our main result, Theorem 3, where we assume that the motion of the base is expansive, it is not necessary to assume that the base chain is simple. It can be any finite embedded graph with straight edges whose relative interiors are pairwise disjoint; a vertex may touch an edge. When the base chain is simple, the results of [9] or [25] guarantee that there is such an expansive motion. On the other hand, although an expansive motion of the base chain of a strictly simple closed polygon to a convex configuration can be guaranteed, it may happen that two realizations are not in the same configuration component, as shown in Fig. 10, and in the conclusion (Sect. 7) there is a description of a case where there are infinitely many components in the configuration space. 
Fig. 4 (a) A half-lens in non-symmetric adornment. (b) A symmetric lens with a point $z$ in the interior of the lens and the adornment
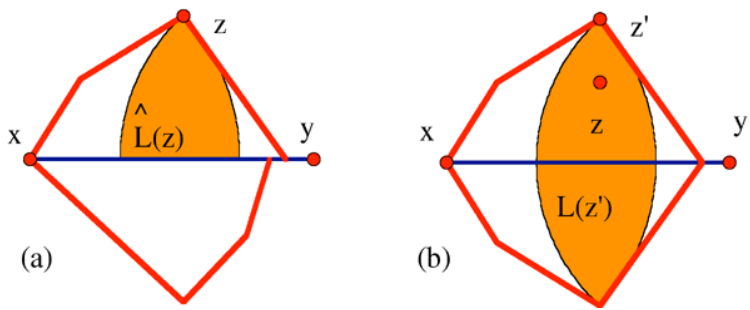

The viewpoint of a chain of shapes as an adorned polygonal chain is useful for two reasons. First, we can more easily talk about the kinds of shapes, and their relation to the locations of the incident hinges, in a family of chains: this information is captured by the adornments. Second, the underlying polygonal chain provides a mechanism for folding the chain of shapes, as well as a natural unfolding goal: straighten the underlying open chain or convexify the underlying closed chain. Indeed, we show that, in some cases, unfolding motions of the polygonal chain induce valid unfolding motions of the chain of shapes.

\subsection{Slender Adornments}

An adornment is defined to be slender if, for a point moving on either side of the shape, the distance to each endpoint of the base changes monotonically (possibly not strictly monotonically). An adornment is called symmetric if it is symmetric about the line through the base. An adornment is called one-sided if it lies in just one of the closed half-planes whose boundary contains the base. Clearly, a general adornment is the union of two one-sided adornments, and a one-sided adornment is the intersection of a symmetric adornment with a closed half-plane whose boundary contains the base. For a base segment $[x, y]$ and a point $z$ in the plane, where $\|z-x\| \leq\|y-x\|$ and $\|z-y\| \leq\|x-y\|$, let $L(z)$ be the intersection of the two disks with $z$ on its boundary, centered at $x$ and at $y$. We call $L(z)$ the lens determined by $z$ associated to the base $[x, y]$. A half-lens, denoted as $\hat{L}(z)$, is the intersection of $L(z)$ and the closed half-plane through the base containing $z$. See Fig. 4 for a picture of a half-lens and lens. The following are some simple, but useful, properties of lenses.

Proposition 1 For any point $z$ in a (symmetric) slender adornment $A, \hat{L}(z) \subset A$ $(L(z) \subset A)$.

Proof Let $z$ be a point on the defining boundary of $A$. Since the distance to $x$ along the boundary is monotone, no point along the path from $z$ to $y$ intersects the interior of the circle centered at $x$ through $z$. Similarly, no point along the path from $z$ to $x$ intersects the interior of the circle centered at $y$ through $z$. Thus, the intersection of the circular disks centered at $x$ and $y$, with $z$ on their boundary, and the closed half-plane containing $z, \hat{L}(z)$ is contained in the adornment. In the symmetric case, the intersection of the circular disks with $z$ on their boundary $L(z)$ is contained in $A$. See Fig. 4.

Proposition 2 For any point $z$ in the interior of a (symmetric) slender adornment $A$, there is a half-lens $\hat{L}\left(z^{\prime}\right) \subset A$ (lens $\left.L\left(z^{\prime}\right) \subset A\right)$ that has $z$ in its interior. 
Proof The half-lens (lens) through $z$ is contained in $A$ by Proposition 1. Since $z$ is in the interior of $A$, there is another point $z^{\prime}$ in $A$ on the line perpendicular to the base segment slightly further away from the base. Then $z$ is in the interior of the half-lens (lens) defined by $z^{\prime}$.

Proposition 3 A symmetric adornment $A$ of a base $[x, y]$ is slender if and only if it is the union of the intersection of pairs of disks centered at $x$ and $y$.

Proof Assume that $A$ is a slender adornment. By Proposition 1, the union of the lenses $L(z)$ for $z$ on the boundary of $A$ is contained in $A$.

To show the reverse containment, any point $z$ in the interior of $A$ lies on a circle centered at $x$, and this circle must intersect the boundary of $A$ in (at least) one point $z^{\prime}$. Then $z$ is in $L\left(z^{\prime}\right)$. Thus, the union of the lenses $L\left(z^{\prime}\right)$ for $z^{\prime}$ on the boundary of the slender adornment contains $A$.

For the converse implication, assume that we have a symmetric adornment $A$ which is formed as the union of lenses, as stated in the proposition. We first show that for two points $z$ and $z^{\prime}$ on the boundary of $A$, we cannot have $\|z-x\|<\left\|z^{\prime}-x\right\|$ and $\|z-y\|<\left\|z^{\prime}-y\right\|$. If this were the case, some lens on whose boundary $z^{\prime}$ lies would contain $z$ in its interior, a contradiction. Since we can exchange the roles of $z$ and $z^{\prime}$, we conclude

$$
\begin{aligned}
& \|z-x\|<\left\|z^{\prime}-x\right\| \quad \Longrightarrow \quad\|z-y\| \geq\left\|z^{\prime}-y\right\|, \quad \text { and } \\
& \|z-x\|>\left\|z^{\prime}-x\right\| \quad \Longrightarrow \quad\|z-y\| \leq\left\|z^{\prime}-y\right\| .
\end{aligned}
$$

Now, if we order the points $z$ on one boundary chain from $x$ to $y$ by the quantity $\|z-x\|-\|z-y\|$, we conclude that the distance $\|z-x\|$ cannot decrease and the distance $\|z-y\|$ cannot increase; otherwise we would derive a contradiction to (1).

Proposition 4 Finite unions and arbitrary intersections of slender adornments are slender adornments.

Proof This follows from Proposition 3 in the symmetric case, and the nonsymmetric case follows from the symmetric case by intersecting with the closed half-plane containing the line segment.

Proposition 5 Every slender adornment is contained in the symmetric lens determined by either of the points equidistant from the endpoints of the base as in Fig. 5.

Proof Any slender adornment must be contained in the disk through the other end of the base, and thus it is in the intersection of those two disks.

\subsection{Alternate Definitions of Slender Adornments}

In the preliminary conference version of this paper [7], we used a more restricted definition of slenderness: suppose that the boundary of an adornment consists of two 
Fig. 5 The largest slender adornment with a given base is a lens $L(z)$, where $\|z-x\|=\|z-y\|=\|y-x\|$

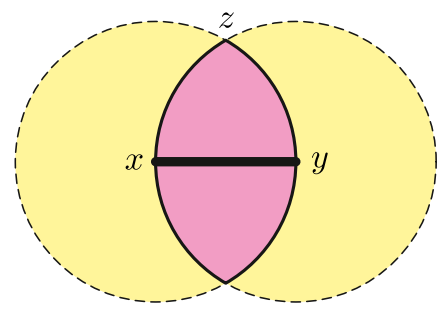

Fig. 6 The normal property for slender shapes

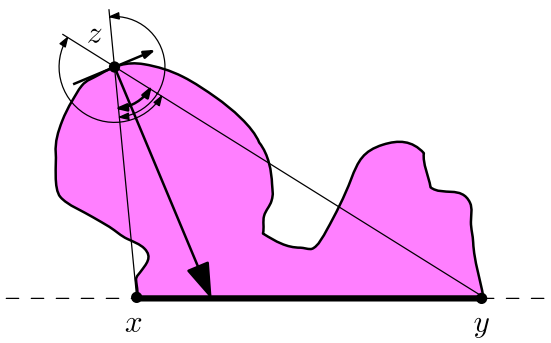

differentiable curves between the base points. Then the condition of being slender is equivalent to requiring that every inward normal of the shape intersects the base before exiting the shape, as illustrated in Fig. 6. This property ensures that slender adornments will not get closer together during an expansive motion of the base. Our current slenderness definition by the monotone distance property is more general, easier to handle, and it does not raise questions of differentiability.

\subsection{Kirszbraun's Theorem}

In what follows it is very handy to have the following theorem of Kirszbraun [21].

Theorem 1 Suppose a finite set of closed circular disks in Euclidean space is rearranged so that no pair of centers gets strictly closer together. If the original set has an empty intersection, so does the rearranged set.

There is a discussion and proof of this in [4] as well as references to other proofs. We only need this result for four disks in the Euclidean plane.

\section{Expanded Slender Symmetric Adornments Never Overlap}

We first prove the following for the case of symmetric slender adornments. Note that the following result is for discrete expansions of the base chain. Recall that two adornments overlap if a point in one adornment lies in the interior of the other. This allows their boundaries to touch, but not to penetrate each other. Note that the bases of a chain do not cross as well, by the expansive property of a discrete motion. We do not need the continuous expansive property for this result. 


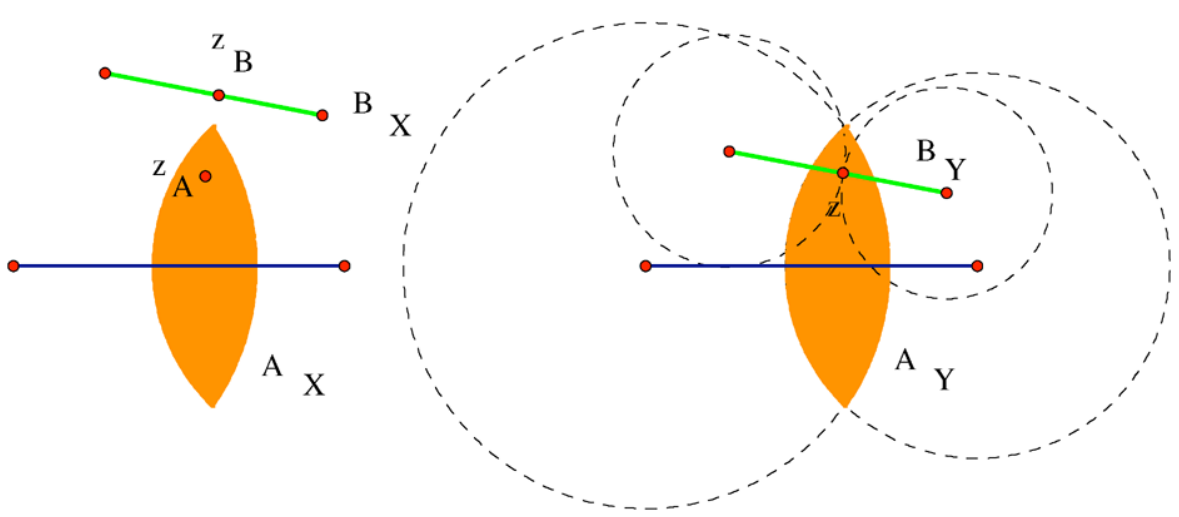

Fig. 7 This is the situation when two adornments overlap. The four circles that used in the application of Kirszbraun's Theorem are indicated. Note that, in this figure, the motion from $X$ to $Y$ is not an expansion, since that would contradict Theorem 2

Theorem 2 Consider two configurations $X$ and $Y$ of corresponding chains with symmetric slender adornments such that the base chain of $Y$ is an expansion of the base chain of $X$. We assume that the adornments attached to the base chain of $X$ do not overlap. Then, when the corresponding adornments are attached to the base chain of $Y$, they also do not overlap.

Proof Suppose $A_{X}$ and $B_{X}$ are two slender adornments attached to different links of the base chain of $X$, and $A_{X}$ and $B_{X}$ do not overlap. Let $A_{Y}$ and $B_{Y}$ be the corresponding adornments for $Y$. Suppose that $z$ is a point in the intersection $A_{Y} \cap B_{Y}$, where $z$ is in the interior of, say, $A_{Y}$. We wish to find a contradiction.

Let $z_{A}$ and $z_{B}$ be the corresponding distinct points in $A_{X}$ and $B_{X}$, respectively, that map to $z$ under the expanding map of their bases. Thus, the lenses $L_{A}\left(z_{A}\right)$ and $L_{B}\left(z_{B}\right)$ for $A_{X}$ and $B_{X}$ have disjoint interiors, since the adornments do not overlap. Since $z$ is in the interior of $A_{Y}$, we can assume that $L_{A}\left(z_{A}\right)$ can be chosen so that the closed lenses $L_{A}\left(z_{A}\right)$ and $L_{B}\left(z_{B}\right)$ are disjoint also. Thus the four circular disks that correspond to the circular disks that define $L_{A}\left(z_{A}\right)$ and $L_{B}\left(z_{B}\right)$ have an empty intersection. By Kirszbraun's Theorem 1 and the expansion property of the endpoints of the bases of $A_{X}$ and $B_{X}$, which are the centers of the four circular disks, the intersection of the corresponding lenses for $A_{Y}$ and $B_{Y}$ must also be empty, contradicting the assumption that $A_{Y}$ and $B_{Y}$ overlap. See Fig. 7.

For discrete expansions, it is not possible to deal with nonsymmetric adornments. Figure 8 shows an example of two chains with corresponding slender adornments, one an expansion of the other. One starts with no overlap, and the other has such an overlap.

\section{Slender Adornments Cannot Lock}

We now consider the general case, assuming a continuous expansive motion. 


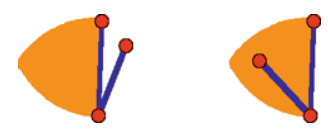

Fig. 8 This shows two chains, with slender but not symmetric adornments, where one is an expansion of the other, while there is an overlap in the expanded configuration, but not the original

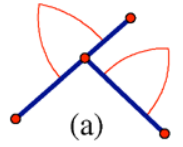

(a)

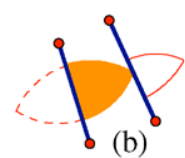

(b)

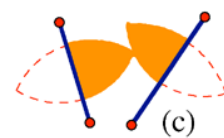

(c)

Fig. 9 This presents the cases when one adornment with its base might start to overlap with the other. The dashed lines indicate where one or both of the lens of the adornment can be extended so that it does not intersect the relevant part of the other. The thick lines indicate the part of the adornment that is not to be penetrated by the other lens or base. The thin lines indicate where some of the rest of the adornment might lie, containing the point $z_{A}$, say, in the proof

Theorem 3 Suppose there is a continuous expansive motion of the base chain with slender non-overlapping, not necessarily symmetric, adornments attached. Then the adornments never overlap during the motion.

Proof Because of the expansive property, two segments of the base chain can only intersect at common endpoints of adjacent segments. Thus, suppose that $z_{A}$, in the interior of adornment $A$, intersects $z_{B}$ in adornment $B$ at some time $t_{1}$ during the motion. We look for a contradiction. By Proposition 2, there is a closed half-lens $L_{A}$ for $A$ that contains $z_{A}$ in its interior, and there is a first time $t_{0}<t_{1}$ when $L_{A}$ intersects another half-lens $L_{B}$ for $B$ that contains $z_{B}$. Necessarily, that intersection must be on the common boundary of $L_{A}$ and $L_{B}$. (Note that $L_{B}$ could be a single point on a base segment.) Then there are three cases that can occur. In each case, we will show that when the motion is continued from $t_{0}$ to $t_{1}, z_{A}$ and $z_{B}$ cannot intersect.

Case 1: The bases of $A$ and $B$ intersect in the interior of at least one of the bases. This cannot happen because the bases are initially disjoint and the motion is expansive. See Fig. 9(a).

Case 2: The base of $A$ or $B$ intersects the half-lens of the other. The half lens can be extended to a full symmetric lens without overlapping the base of the other. Applying Theorem 2, we see that $z_{A}$ and $z_{B}$ cannot intersect upon further expansion. See Fig. 9(b).

Case 3: The half lenses of $A$ and $B$ intersect. In this case both half lenses can be extended to nonoverlapping symmetric lenses. Again we apply Theorem 2 to see that $z_{A}$ and $z_{B}$ cannot intersect upon further expansion. See Fig. 9(c).

Corollary 4 A strictly simple polygonal chain with slender adornments attached can always be straightened or convexified by a continuous motion.

Proof By [9], there is a continuous expansive motion of the base chain, where the final configuration is convex in the case of a closed chain and straight in the case 
(a)

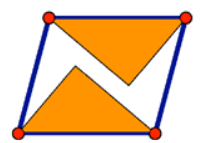

(b)

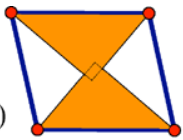

(c)

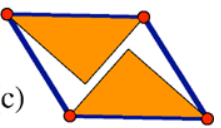

Fig. 10 Two configurations (a) and (c) of a quadrilateral with two slender adornments attached. It is not possible to continuously move from one to the other without colliding. Part (b) shows how the two adornments collide as the quadrilateral is deformed from $(\mathbf{a})$ to $(\mathbf{c})$

Fig. 11 The base chain of part (a) expands to part (b). But the dark points on the corresponding slender adornments get closer together
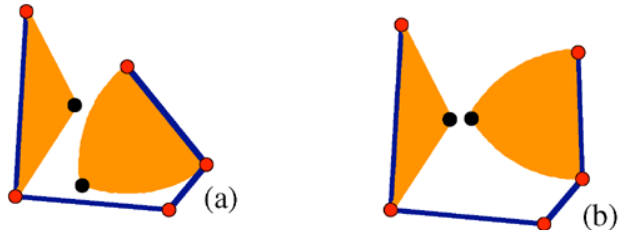

of an open chain. Then Theorem 3 implies that they can be carried along without overlap.

Corollary 5 A strictly simple open polygonal chain with slender adornments can be continuously reconfigured between any two states.

Proof By Corollary 4, both chains can be continuously expansively reconfigured so that the base chains are straight. Thus, one state can be expanded to have a straight base configuration and then contracted to the other configuration by running its expansion backwards.

It is interesting to note that the conclusion of Corollary 5 does not hold for closed chains, even though any two convex chains with no adornments can be continuously reconfigured from one to the other. Figure 10 shows an example where the configuration space has two components, where the base chain is a quadrilateral, and where each adornment is a triangle attached to its base.

Indeed, in the conclusion (Sect. 7) it is shown how to create a quadrilateral with two slender adornments such that the configuration space has infinitely many components.

It is also interesting to note that when the base chain is expanded, it often happens that the motion on the adorned configuration is not expansive. Figure 11 shows an example.

\section{Generalizations: Overlapping Adornments and Generalized Slender Symmetric Adornments}

In the discussion so far, we have assumed, when the adornments are attached to their chains, that they do not overlap. What happens when the slender adornments do overlap? It turns out that we can apply some of the results of [6] related to problems concerning areas of unions and intersections of circular disks in the plane to the case where the adornments are all symmetric. 
Fig. 12 Three intervals with overlapping symmetric slender adornments
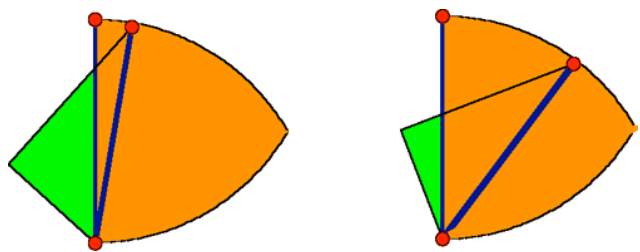

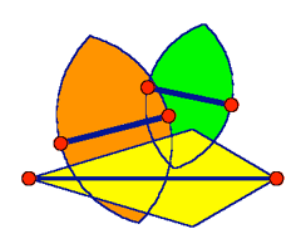

Fig. 13 An example of two chains with non-symmetric slender adornments, where the expanded chain with adornments has smaller area 
Fig. 14 A locked chain of nine equilateral triangles. (a) Drawn loosely. Separations should be smaller than they appear. (b) Drawn tightly, with no separation, as a self-touching configuration

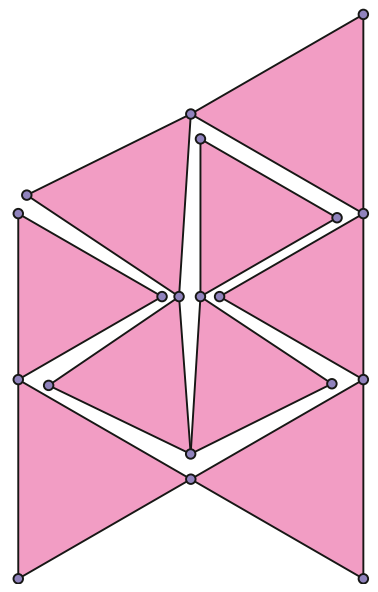

(a)

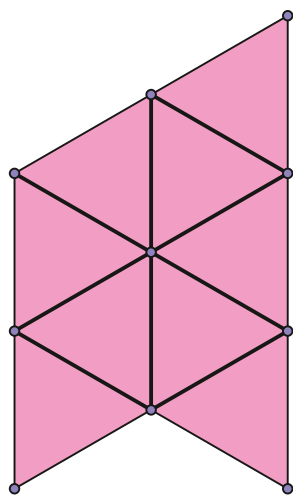

(b)

Another possible generalization is to attach the analog of slender adornments to simplicial complexes in higher dimensions. For example, a set $A$ in three-space would be called slender with respect to a triangle base $B$ if for any plane $P$ perpendicular to the plane of $A, P \cap A$ is slender with respect to $B \cap A$. Then the analog of Theorem 3 should hold using the notion of symmetric slender adornments. Even the analog of Theorem 7 for the volumes of symmetric slender adornments would still hold, but it could only be asserted for continuous expansions of the base chain. The higherdimensional version of Corollary 8 in [6], on which Lemma 6 is based, is not known for discrete expansions. However, in [13], there is a continuous version that will suffice. In higher dimensions, the idea is to assume simply that the base chain, to which the adornments are attached, is expanded.

\section{Locked Chains of Sharp Triangles}

An isosceles triangle with an apex angle of $\geq 90^{\circ}$ and with the nonequal side as the base is a slender adornment. By Corollary 4, any chain of such triangles can be straightened. In this section we show that this result is tight: for any isosceles triangle with an apex angle of $<90^{\circ}$ and with the nonequal side as a base, there is a chain of these triangles that cannot be straightened.

Figure 14(a) shows the construction for equilateral triangles (of slightly different sizes). This figure is drawn with the pieces loosely separated, but the actual construction has arbitrarily small separations and arbitrarily closely approximates the selftouching geometry shown in Fig. 14(b). Stretching the triangles in this self-touching geometry, as shown in Fig. 15, defines our construction for any isosceles triangles with an opposite angle of any value less than $90^{\circ}$. In this case, however, our construction uses two different scalings of the same triangle. 
Fig. 15 Variations on the self-touching configuration from Fig. 14(b) to have any desired angle $<90^{\circ}$ opposite the base of each triangle
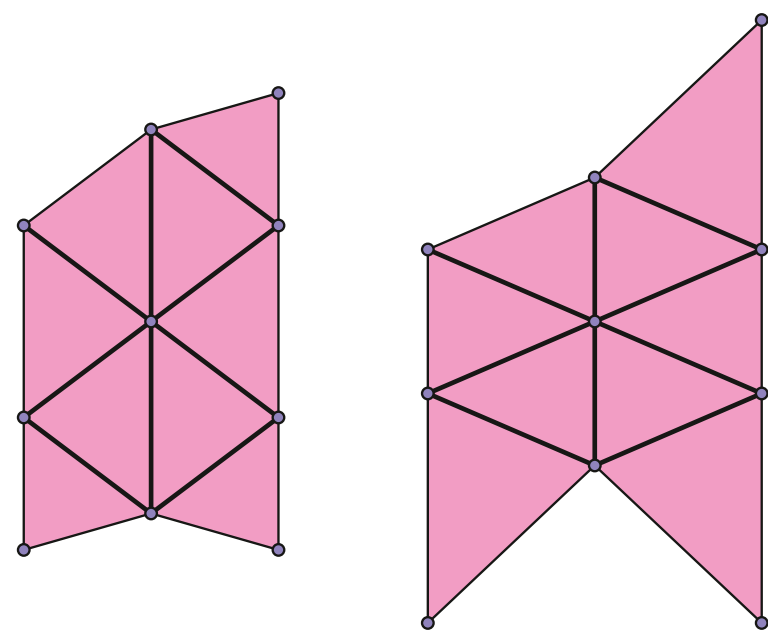

\subsection{Theory of Self-touching Configurations}

This view of the construction as a slightly separated version of a self-touching configuration allows us to apply the program developed in [8] for proving a configuration locked. This theory allows us to study the rigidity of self-touching configurations, which is easier because vertices cannot move even slightly, and obtain a strong form of lockedness of non-self-touching perturbations drawn with sufficiently small (but positive) separations.

To state this relation precisely, we need some terminology from [8]. Call a linkage configuration rigid if it cannot move at all. Define a $\delta$-perturbation of a linkage configuration to be a repositioning of each vertex within distance $\delta$ of its original position, without regard to preserving edge lengths (better than $\pm 2 \delta$ ), but consistent with the combinatorial information of which vertices are on which side of which bar. Call a linkage locked within $\epsilon$ if no motion that leaves some bar pinned to the plane moves any point by more than $\epsilon$. Call a self-touching linkage configuration strongly locked if, for any desired $\epsilon>0$, there is a $\delta>0$ such that all $\delta$-perturbations are locked within $\epsilon$. Thus, if a self-touching configuration is strongly locked, then the smaller we draw the separations in a non-self-touching perturbation, the less the configuration can move. In particular, if we choose $\epsilon$ small enough, the linkage must be locked in the standard sense of having a disconnected configuration space locally.

Theorem 8 [8, Theorem 8.1] If a self-touching linkage configuration is rigid, then it is strongly locked.

Therefore, if we can prove that the self-touching configuration in Fig. 14(b) (and its variations in Fig. 15) are rigid, then sufficiently small perturbations along the lines shown in Fig. 14(a) are rigid.

The theory of [8] also provides tools for proving rigidity of a self-touching configuration. Specifically, we can study infinitesimal motions, which just define the begin- 
Fig. 16 Two zero-length connections between vertices $u$ and $v$

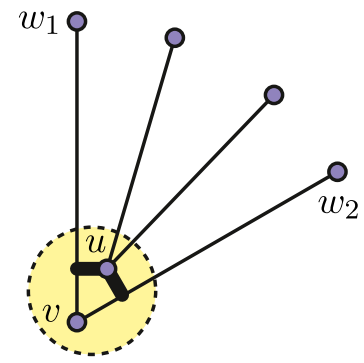

ning of a motion to the first order. Call a configuration infinitesimally rigid if it has no infinitesimal motions.

Lemma 9 [8, Lemma 6.1] If a self-touching linkage configuration is infinitesimally rigid, then it is rigid.

A final tool we need from [8] is for proving infinitesimal rigidity. For each vertex $u$ wedged into a convex angle between two bars $\left\{v, w_{1}\right\}$ and $\left\{v, w_{2}\right\}$, we say that there are two so-called zero-length connections between $u$ and $v$, one perpendicular to each of the two bars $\left\{v, w_{i}\right\}$. Such a connection between a point $u$ and a bar $\{v, w\}$ restricts $u$ to one side of the line through $v, w,{ }^{1}$ see Fig. 16. These connections must increase to the first order because $u$ must not cross the two bars $\left\{v, w_{i}\right\}$. In proving infinitesimal rigidity, we can choose to discard any zero-length connections we wish, because ignoring some of the noncrossing constraints only makes the configuration more flexible. Together, the bars and the zero-length connections are the edges of the configuration. Define a stress to be an assignment of real numbers (stresses) to edges such that, for each vertex $v$, the vectors with directions defined by the edges incident to $v$, and with magnitudes equal to the corresponding stresses, sum to the zero vector. We denote the stress on a bar $\{v, w\}$ by $\omega_{v w}$, and we denote the stress on a zero-length connection between vertex $u$ and vertex $v$ perpendicular to $\{v, w\}$ by $\omega_{u, v w}$.

Lemma 10 [8, Lemma 7.2] If a self-touching configuration has a stress that is negative on every zero-length connection and if the configuration is infinitesimally rigid when every zero-length connection is treated as a bar pinning two vertices together, then the self-touching configuration is infinitesimally rigid.

\subsection{Locked Chains}

We are now in the position to state the precise senses in which the chains of isosceles triangles in Figs. 14 and 15 are locked:

\footnotetext{
${ }^{1}$ The definition of zero-length connections in [8] is more general, but this definition suffices for our purposes. The term "zero-length connection" is perhaps unfortunate since it mistakenly suggests a constraint on the distance between $u$ and $v$ to be zero. The zero-length connection is, however, allowed to slide freely on the bar $\{v, w\}$. 
Fig. 17 Rule 1 for simplifying self-touching configurations

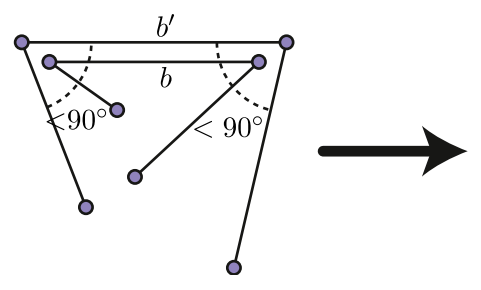

Theorem 11 The self-touching chains of nine isosceles triangles shown in Figs. 14(b) and 15 are rigid provided that the apex angle is $<90^{\circ}$.

Applying Theorem 8, we obtain the desired result:

Corollary 12 The self-touching chains of nine isosceles triangles shown in Figs. 14(b) and 15 are strongly locked, provided that the apex angle is $<90^{\circ}$. Therefore, any sufficiently small non-self-touching perturbation, similar to the one shown in Fig. 14(a), is locked.

Sections 6.3-6.4 prove Theorem 11.

\subsection{Simplifying Rules}

We introduce two obvious rules that significantly restrict the allowable motions of the self-touching configuration of isosceles triangles.

Rule 1 If $a$ bar $b$ is collocated with another bar $b^{\prime}$ of equal length, and the bars incident to $b^{\prime}$ form angles less than $90^{\circ}$ on the same side as $b$, then any motion must keep $b$ collocated with $b^{\prime}$ for some positive time. See Fig. 17.

Proof The noncrossing constraints at the endpoints of $b$ and $b^{\prime}$ prevent $b$ from moving relative to $b^{\prime}$ until the angles at the endpoints of $b^{\prime}$ open to $\geq 90^{\circ}$, which can only happen after a positive amount of time.

More formally, suppose without generality of loss that the endpoints of $b$ and $b^{\prime}$ are initially $(-1,0)$ and $(1,0)$ with $b$ below $b^{\prime}$ as in Fig. 17 . Let $\alpha, \beta<90^{\circ}$ denote the angles at the endpoints of $b^{\prime}$. We attach the coordinate system to $b^{\prime}$ and denote by $(-1+x, y)$ and $(1+u, v)$ the (moving) endpoints of $b$. Then we have

$$
y \leq 0, \quad v \leq 0, \quad y \geq-x \tan \alpha, \quad v \geq u \tan \beta,
$$

and

$$
((x-1)-(1+u))^{2}+(y-v)^{2}=4 .
$$

From (3) we conclude that $x \geq 0, u \leq 0$, and hence $x-u \geq 0$, and furthermore

$$
0 \leq-y-v \leq d(x-u)
$$


Fig. 18 Applying Rule 1 to the chain of nine equilateral triangles from Fig. 14
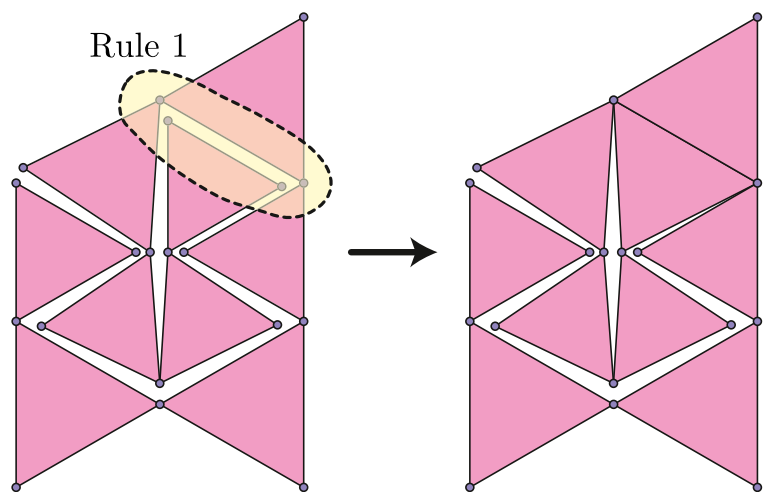

Fig. 19 Rule 2 for simplifying self-touching configurations
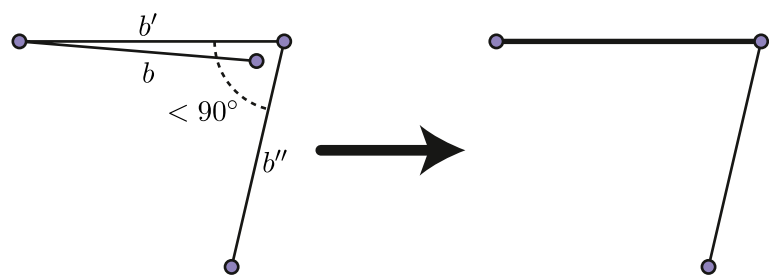

with $d=\max \{\tan \alpha, \tan \beta\} \geq 0$. From (4) we obtain

$$
\begin{aligned}
(x-u)^{2}-4(x-u) & =-(y-v)^{2} \geq-(y-v)^{2}-4 y v=-(-y-v)^{2} \\
& \geq-d^{2}(x-u)^{2} .
\end{aligned}
$$

The last inequality is based on (5). Now if $x-u \neq 0$, we can divide by $x-u$, knowing from (5) that $x-u>0$, and obtain $x-u \geq 4 /\left(1+d^{2}\right)$. Since $x$ and $u$ have to move continuously from their starting values $x=u=0$, this is impossible. We conclude that $x-u=0$, and hence $x=u=0$. Substituting this into (6), we see that $-(-y-v)^{2}=0$ is sandwiched between two expressions which are 0 . Therefore $-y-v=0$, and hence $y=v=0$.

We can apply this rule to the region shown in Fig. 18, resulting in a simpler linkage with the same infinitesimal behavior. Although the figure shows positive separations for visual clarity, we are in fact acting on the self-touching configuration of Fig. 14(b).

Rule 2 If a bar b is collocated with an incident bar $b^{\prime}$ of the same length whose other incident bar $b^{\prime \prime}$ forms a convex angle with $b^{\prime}$ surrounding $b$, then any motion must keep $b$ collocated with $b^{\prime}$ for some positive time. See Fig. 19.

Proof The noncrossing constraints at the endpoint of $b$ surrounded by the convex angle formed by $b^{\prime}$ and $b^{\prime \prime}$ prevent $b$ from moving relative to $b^{\prime}$ until the convex angle opens to $\geq 90^{\circ}$, which can only happen after a positive amount of time. The formal proof is similar (and simpler) as for Rule 1. 

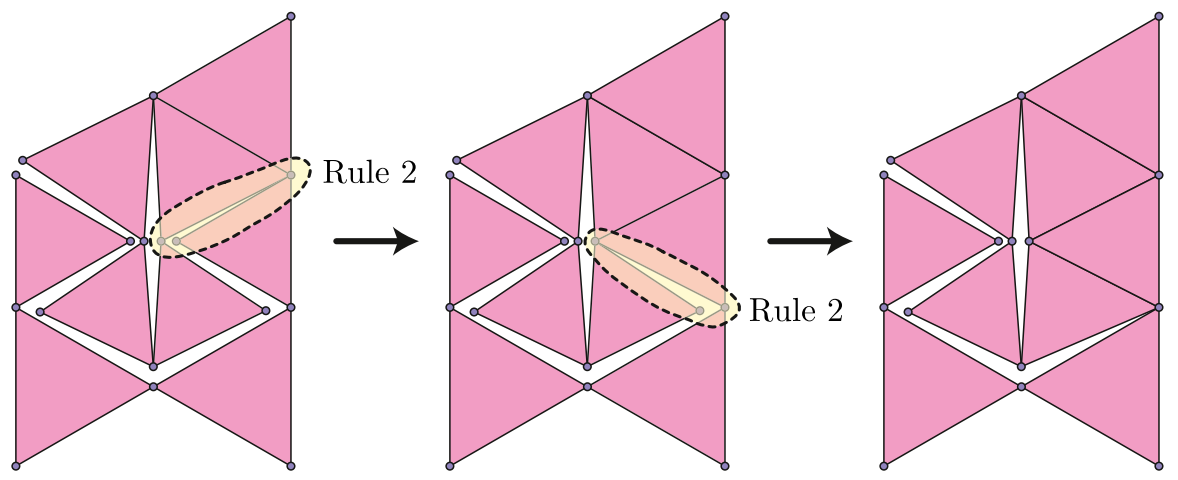

Fig. 20 Applying Rule 2 twice to the configuration from Fig. 18

Fig. 21 The simplified configuration from Fig. 20

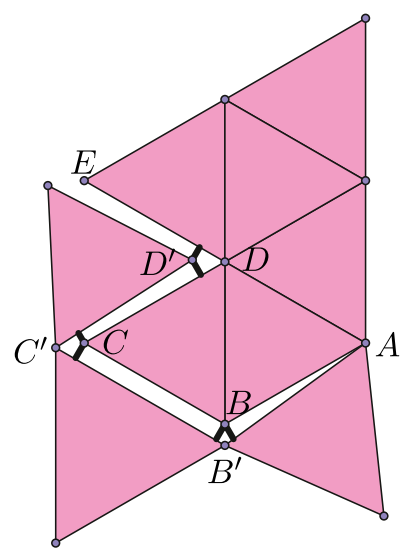

We can apply this rule twice, as shown in Fig. 20, to further simplify the linkage.

The final simplification comes from realizing that the central quadrangle gap between triangles is effectively a triangle because the right pair of edges are a rigid unit. Thus the gap forms a rigid linkage (though it is not infinitesimally rigid, because a horizontal movement of the central vertex would maintain distances to the first order), so we can treat it as part of a large rigid block. Figure 21 shows a simplified drawing of this self-touching configuration, which is rigid if and only if the original self-touching configuration is rigid.

\subsection{Stress Argument}

Finally we argue that the simplified configuration of Fig. 21 is infinitesimally rigid using Lemma 10. The configuration is clearly infinitesimally rigid if $B$ is pinned against $B^{\prime}, C$ is pinned against $C^{\prime}$, and $D$ is pinned against $D^{\prime}$. It remains to construct a stress that is negative on all length-zero connections. The stress we construct is nonzero only on the edges connecting points with labels in Fig. 21; we also set $\omega_{A D}=0$. 
We start by assigning the stresses incident to $A$. We choose $\omega_{A B}<0$ arbitrarily and set $\omega_{A B^{\prime}}:=-\omega_{A B}>0$. $A$ is now in equilibrium because these stress directions are parallel.

We symmetrically assign $\omega_{B C}:=\omega_{A B}<0$ and $\omega_{B^{\prime} C^{\prime}}=\omega_{A^{\prime} B^{\prime}}>0$. The resulting forces on $B$ and $B^{\prime}$ are vertical. They can be balanced by an appropriate choice of the stresses $\omega_{B, B^{\prime} A}=\omega_{B, B^{\prime} C^{\prime}}<0$, which, taken together, also point in the vertical direction.

Vertex $D^{\prime}$ has exactly three incident stresses- $-\omega_{C^{\prime} D^{\prime}}, \omega_{D^{\prime}, D C}$, and $\omega_{D^{\prime}, D E}-$ which do not lie in a halfplane. Thus there is an equilibrium assignment to these stresses, unique up to scaling, and the stresses all have the same sign. Because zerolength connections must be negative, we are forced to make all three of these stresses negative. We also choose this scale factor to be substantially smaller than the stresses that have been assigned so far.

By assigning $\omega_{C D}=-\omega_{C^{\prime} D^{\prime}}$, we establish equilibrium at vertex $D$ as well: the forces at $D$ are the same as at $D^{\prime}$, only with reversed signs.

Vertex $C$ feels two stresses assigned so far $-\omega_{C D}>0$ and $\omega_{B C}<0$. By the choice of scale factors, the latter force dominates, leaving us with a negative force in the direction close to $C B$, and two stresses $\omega_{C, C^{\prime} B^{\prime}}$ and $\omega_{C, C^{\prime} D^{\prime}}$ which can be used to balance this force. The three directions do not lie in a halfplane. Therefore $\omega_{C, C^{\prime} B^{\prime}}$ and $\omega_{C, C^{\prime} D^{\prime}}$ can be assigned negative stresses.

Finally, vertex $C^{\prime}$ is also in equilibrium because $\omega_{B^{\prime} C^{\prime}}=-\omega_{B C}, \omega_{C^{\prime} D^{\prime}}=-\omega_{C D}$, and the stresses from the zero-length connections are the same as for $C$ but in the opposite direction.

In summary, we have shown the existence of a stress that is positive on all zerolength connections. By Lemma 10, the self-touching configuration is infinitesimally rigid; so by Lemma 9, the configuration is rigid. By the simplification arguments above, the original self-touching configuration is also rigid. By Theorem 8 , the original self-touching configuration is strongly locked, so sufficiently perturbations are locked.

We remark that an argument similar to the one above, using an assignment of stresses, can also be used for proving Rules 1 and 2, with an appropriate modification of Lemma 10; however, the direct argument that we have given is simpler.

The argument relied on the isosceles triangles having an apex angle of $<90^{\circ}$ (but no more) in order to guarantee that particular triples of stress directions are or are not in a halfplane. It also relies on the symmetry of the configuration through a vertical line (excluding the triangle in the upper right). Thus the argument generalizes to all isosceles triangles sharper than $90^{\circ}$.

\subsection{Locked Equilateral Triangles}

Figure 22 shows another, simpler example of a locked chain of equilateral triangles, using just seven triangles instead of nine. However, this example cannot be stretched into a locked chain of triangles with an arbitrary apex angle of $<90^{\circ}$, as in Fig. 15.

To prove that this example is locked, we first apply Rule 1 and then Rule 2, as shown in Fig. 23. Unlike the previous example, the resulting simplified configuration is not infinitesimally rigid (the middle vertex can move infinitesimally horizontally), 

configuration

Fig. 22 A locked chain of seven equilateral triangles. (a) Drawn loosely. Separations should be smaller than they appear. (b) Drawn tightly, with no separation, as a self-touching

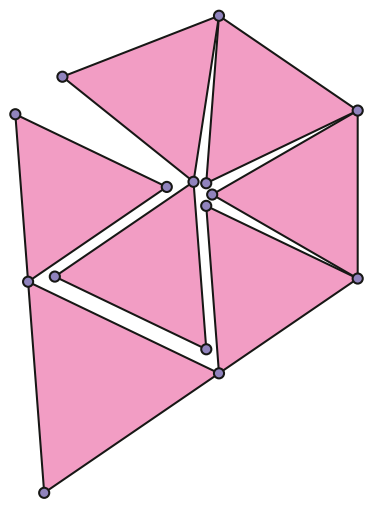

(a)
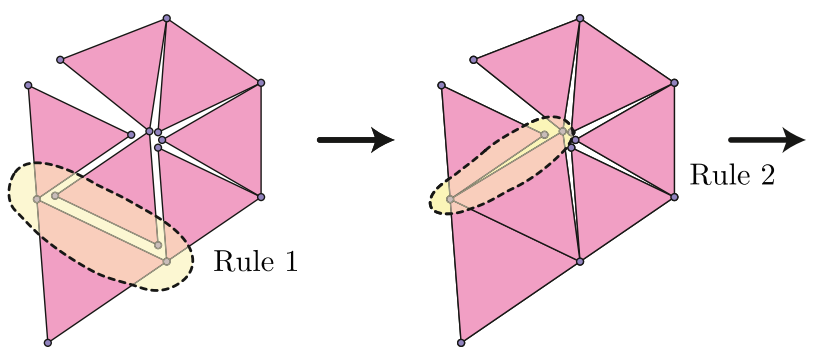

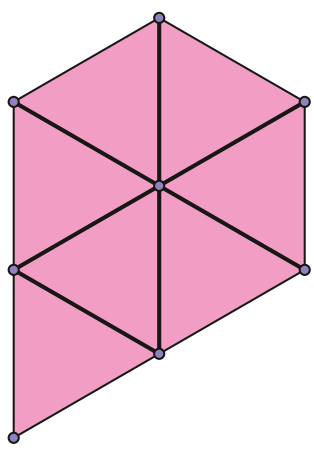

(b)

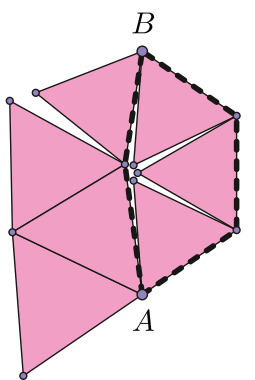

Fig. 23 Applying Rules 1 and 2 to the chain of seven equilateral triangles from Fig. 22

so we cannot use a stress argument. In this case, however, we can use a more direct argument to prove rigidity of the simplified configuration (and thus of the original self-touching configuration).

Let $\ell$ denote the side length of the triangles in any of the self-touching configurations. Consider the two dashed chains connecting vertices $A$ and $B$ in the simplified configuration. The left chain of two bars forces the distance between $A$ and $B$ to be at most $2 \ell$, with equality as in the original configuration only if the angle between the two bars remains straight. The right chain of three bars can only open its angles, because of the three triangles on the inside, so the right chain acts as a Cauchy arm. The Cauchy-Steinitz Arm Lemma (see, e.g., [10] or [26]) proves that the endpoints of such a chain can only get farther away from each other. Thus the distance between $A$ and $B$ is at least $2 \ell$, with equality only if the angles in the right chain do not change. These upper and lower bounds of $2 \ell$ on the distance between $A$ and $B$ force the bounds to hold with equality, which prevents any angles from changing except possibly for the angles at $A$ and $B$. However, it is impossible to change fewer than four angles of a closed chain such as the one formed by the left and right dashed chains. (This simple fact was also proved by Cauchy [12].) Therefore, the configuration is rigid. 
Fig. 24 Chains of squares that appear to be locked: (a) an open chain, and (b) a closed chain. The squares are self-touching; the drawing includes gaps only for clarity of illustration

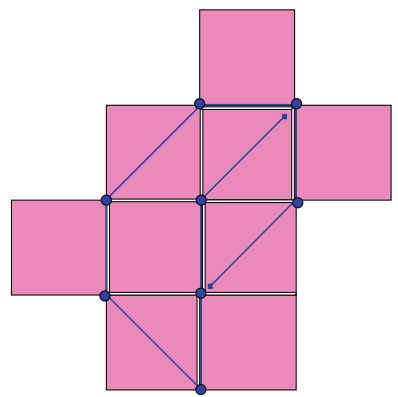

(a)

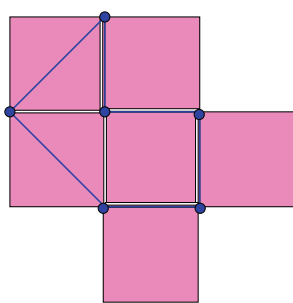

(b)

Applying Theorem 8, we obtain that the self-touching configuration is strongly locked:

Theorem 13 The self-touching chain of seven equilateral triangles shown in Fig. 22(b) is rigid and thus strongly locked. Therefore, any sufficiently small nonself-touching perturbation, similar to the one shown in Fig. 22(a), is locked.

\section{Conclusion}

In the time since we first reported some of our results in the form of an extended abstract [7], a number of further extensions have been explored. Abbott et al. [1] have utilized our results in proving that hinged dissections exist. Abbott, Demaine, and Gassend [2] provided a generalization for the restricted case of open chains of strictly slender adornments: even when self-touching configurations are allowed, every chain can be opened.

A variety of open questions and extensions remain to be studied. It may be interesting to consider the algorithmic question of computing, for a given configuration of an adorned chain (whose adornments are not slender), whether or not there exists a motion that opens it.

Our results have application to hinged dissections of polyregulars, e.g., polyominoes; this includes the (slender) case of squares connected at opposite corners. An interesting question arising in this context is whether every hinged dissection can be subdivided so that the pieces are slender.

We note that there are examples of open and closed chains of self-touching squares (some of which are attached at adjacent corners) that we conjecture to be locked (Fig. 24); while we have been unable so far to prove that they are, the methods we employed for the locked chains of sharp triangles (Sect. 6) may be applicable.

One difficulty of exploring the space of adorned chains is highlighted by the following construction. It consists of a closed chain, a convex parallelogram, with slender adornments attached, where each adornment together with its base is convex, such that the configuration space has infinitely many components. We attach a single obtuse triangle as a slender adornment to the top base segment, as with Fig. 10. If the bottom segment is fixed, the path of the bottom vertex in the upper adornment traces 
Fig. 25 Part (a) shows the overall set-up of a parallelogram with two convex slender adornments attached such that the configuration space has infinitely many components. Part (b) is an exaggerated close-up of where the two adornments are close (a)

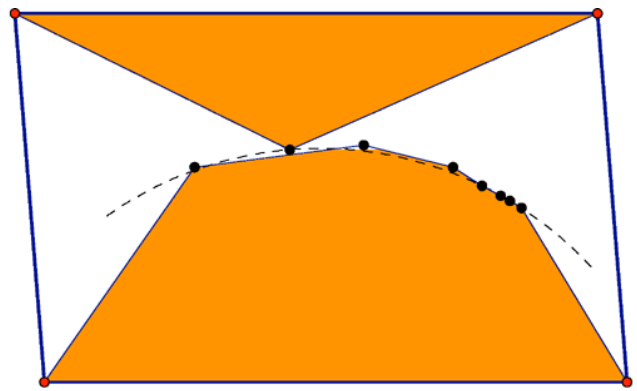

(b)

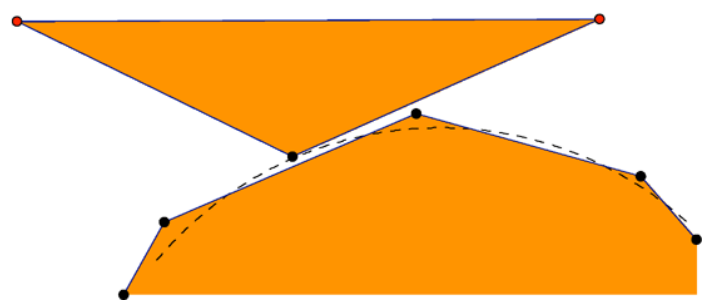

out a circle, which is shown as a dashed circular arc $C$ in Fig. 25(a). The second slender adornment is attached to the bottom segment and is the convex hull of infinitely many points, each slightly above $C$. The points form an infinite sequence $p_{1}, p_{2}, \ldots$ converging to a point on the right $p_{\infty}$, and they are chosen so that the straight line interval from $p_{i}$ to $p_{i+1}$ intersects the lower portion of $C$ (the open circular disk determined by $C$ ). An exaggerated picture of this construction is in Fig. 25(b). Thus, the upper slender adornment intersects the lower adornment and misses it alternately infinitely often.

\section{References}

1. Abbott, T.G., Abel, Z., Charlton, D., Demaine, E.D., Demaine, M.L., Kominers, S.D.: Hinged dissections exist. In: Proceedings of the 24th Annual ACM Symposium on Computational Geometry (SoCG 2008), pp. 110-119, College Park, Maryland, 9-11 June 2008

2. Abbott, T.G., Demaine, E.D., Gassend, B.: A generalized Carpenter's Rule Theorem for self-touching linkages, January 2009. arXiv:0901.1322

3. Alt, H., Knauer, C., Rote, G., Whitesides, S.: On the complexity of the linkage reconfiguration problem. In: Pach, J. (ed.) Towards a Theory of Geometric Graphs. Contemporary Mathematics, vol. 342, pp. 1-14. American Mathematical Society, Providence (2004)

4. Alexander, R.: The circumdisk and its relation to a theorem of Kirszbraun and Valentine. Math. Mag. 57(3), 165-169 (1984)

5. Akiyama, J., Nakamura, G.: Dudeney dissection of polygons. In: Revised Papers from the Japan Conference on Discrete and Computational Geometry. Lecture Notes in Computer Science, vol. 1763, pp. 14-29. Springer, Tokyo (1998)

6. Bezdek, K., Connelly, R.: Pushing disks apart-the Kneser-Poulsen conjecture in the plane. J. Reine Angew. Math. 553, 221-236 (2002)

7. Connelly, R., Demaine, E.D., Demaine, M.L., Fekete, S.P., Langerman, S., Mitchell, J.S.B., Ribó, A., Rote, G.: Locked and unlocked chains of planar shapes. In: Proceedings of the 22nd Annual Symposium on Computational Geometry, Sedona, pp. 61-70. Association for Computing Machinery, New York (2006). arXiv:cs/0604022 
8. Connelly, R., Demaine, E.D., Rote, G.: Infinitesimally locked self-touching linkages with applications to locked trees. In: Calvo, J., Millett, K., Rawdon, E. (eds.) Physical Knots: Knotting, Linking, and Folding of Geometric Objects in 3-space, pp. 287-311. American Mathematical Society, Providence (2002)

9. Connelly, R., Demaine, E.D., Rote, G.: Straightening polygonal arcs and convexifying polygonal cycles. Discrete Comput. Geom. 30(2), 205-239 (2003)

10. Connelly, R.: Rigidity and energy. Invent. Math. 66(1), 11-33 (1982)

11. Connelly, R.: Expansive motions. In: Goodman, J.E., Pach, J., Pollack, R. (eds.) Surveys on Discrete and Computational Geometry-Twenty Years Later. Contemporary Mathematics, vol. 453, pp. 213229. American Mathematical Society, Providence (2008)

12. Cromwell, P.R.: Equality, rigidity, and flexibility. In: Polyhedra, pp. 219-247. Cambridge University Press, Cambridge (1997). Chap. 6

13. Csikós, B.: On the volume of flowers in space forms. Geom. Dedic. 86(1-3), 59-79 (2001)

14. Cheong, J.-S., van der Stappen, A.F., Goldberg, K., Overmars, M.H., Rimon, E.: Immobilizing hinged polygons. Int. J. Comput. Geom. Appl. 17(1), 45-70 (2007)

15. Demaine, E.D., Demaine, M.L., Eppstein, D., Frederickson, G.N., Friedman, E.: Hinged dissection of polyominoes and polyforms. Comput. Geom.: Theory Appl. 31(3), 237-262 (2005)

16. Demaine, E.D., Demaine, M.L., Lindy, J.F., Souvaine, D.L.: Hinged dissection of polypolyhedra. In: Proceedings of the 9th Workshop on Algorithms and Data Structures. Lecture Notes in Computer Science, vol. 3608, pp. 205-217. Waterloo, Canada, August 2005

17. Dudeney, H.E.: Puzzles and prizes. Weekly Dispatch, 1902. The puzzle appeared in the April 6 issue of this column. An unusual discussion followed on April 20, and the solution appeared on May 4

18. Eppstein, D.: Hinged kite mirror dissection, June 2001. arXiv:cs.CG/0106032

19. Frederickson, G.N.: Hinged Dissections: Swinging \& Twisting. Cambridge University Press, Cambridge (2002)

20. Gordon, Y., Meyer, M.: On the volume of unions and intersections of balls in Euclidean space. In: Lindenstrauss, J., Milman, V. (eds.) Geometric Aspects of Functional Analysis. Israel Seminar (GAFA) 1992-1994. Operator Theory: Advances and Applications, vol. 77, pp. 91-101. Birkhäuser, Basel (1995)

21. Kirszbraun, M.D.: Über die zusammenziehende und Lipschitzsche Transformationen. Fundamenta Mathematicae 22, 77-108 (1934)

22. Mao, C., Thallidi, V.R., Wolfe, D.B., Whitesides, S., Whitesides, G.M.: Dissections: Self-assembled aggregates that spontaneously reconfigure their structures when their environment changes. J. Am. Chem. Soc. 124, 14508-14509 (2002)

23. Reif, J.H.: Complexity of the mover's problem and generalizations. In: Proceedings of the 20th Annual IEEE Symposium on Foundations of Computer Science, pp. 421-427, 1979

24. Streinu, I.: A combinatorial approach to planar non-colliding robot arm motion planning. In: Proceedings of the 41st Annual Symposium on Foundations of Computer Science, pp. 443-453, Redondo Beach, California, 2000

25. Streinu, I.: Pseudo-triangulations, rigidity and motion planning. Discrete Comput. Geom. 34(4), 587635 (2005)

26. Schoenberg, I.J., Zaremba, S.K.: On Cauchy's lemma concerning convex polygons. Can. J. Math. 19(4), 1062-1071 (1967) 